\title{
Análisis de las actitudes de estudiantes de traducción hacia las herramientas TAO. El punto de vista de los estudiantes de chino-español
}

\section{Analysis of Translation Students' Attitudes towards CAT Tools. The Point of View of Chinese-Spanish Students}

\section{Carmen VALERO-Garcés y Yanping TAN}

Universidad de Alcalá

carmen.valero@uah.es / yanping.tan@uah.es

Recibido: marzo-2020. Revisado: abril-2020. Aceptado: junio-2020.

Resumen: En el siglo XXI la traducción y las innovaciones tecnológicas son ya inseparables. Las herramientas de traducción asistida por ordenador (herramientas TAO) y la traducción automática (TA) son cada vez más importantes e indispensables en la formación de los futuros traductores. El objetivo principal de este estudio es analizar las actitudes de estos estudiantes hacia las tecnologías de traducción. Específicamente, este trabajo pretende hacer un primer acercamiento a las opiniones y actitudes de los estudiantes chinos sobre las herramientas de TAO y TA en la combinación chinoespañol.

Palabras clave: Herramientas TAO; traducción automática (TA); actitudes de estudiantes; chino-español.

Carmen VALERO-GARCÉS y Yanping TAN Análisis de las actitudes de estudiantes de traducción hacia las herramientas TAO... 


\begin{abstract}
In the 21st century, translation and technological innovations are already inseparable. Computer-assisted translation tools (CAT tools) and machine translation (MT) are increasingly important and essential in the training of future translators. The main objective of this article is to analyze the attitudes of students towards translation technologies. Specifically, this work aims to make a first approach to the opinions and attitudes of Chinese students about CAT tools and AT in the Chinese-Spanish combination.
\end{abstract}

Keywords: CAT tools; machine translation (TAT); students' attitude; Chinese-Spanish.

\title{
1. INTRODUCCIÓN
}

Las herramientas de traducción asistida por ordenador (herramientas TAO) y la traducción automática (TA) son cada vez más importantes, y casi indispensables, no solo en la rutina de trabajo del traductor, sino también en nuestra vida cotidiana. Son también cada vez más frecuentes los estudios que toman en consideración los puntos de vista de los traductores profesionales y de los proveedores de servicios lingüísticos (PSL) sobre el uso e influencia de estas tecnologías en el mercado de trabajo (Çetiner 2018; EUATC 2019).

Las lenguas europeas -y sobre todo el inglés en su carrera por convertirse en lingua franca - suelen ser las lenguas de trabajo y de referencia. En consecuencia, los estudios que tratan de la aceptación de estas herramientas por parte de los estudiantes de traducción o que investigan sus actitudes hacia estas herramientas por lo general se refieren a los hablantes de estas lenguas. Por el contrario, son muy limitados los estudios que tratan con hablantes de lenguas no europeas, pero con una alta presencia en la UE (p. ej. chino, árabe).

A su vez, crece el consenso sobre el hecho de que los futuros profesionales deben contar con unas sólidas destrezas en el uso de las nuevas tecnologías. Por otro lado, como indica Díaz Fouces $(2019,77)$, este proceso de inclusión continuará avanzando de un modo si cabe más acelerado, dado que los cambios tecnológicos tienden a ser invasivos y exponenciales. Parece claro que la formación de traductores debería estar orientada a enseñarles no solo a hacer sino, al mismo tiempo, a comprender. En definitiva, resulta necesario proporcionar a los formadores de traductores un conjunto de herramientas, conceptuales y prácticas, para ayudarles a formar unos profesionales plenamente cualificados, en lugar de (únicamente) unos trabajadores más eficientes (Díaz Fouces 2019, 77).

En esa línea, el objetivo principal de este estudio es analizar las actitudes de los estudiantes hacia las tecnologías de traducción. Específicamente, este trabajo pretende ser un primer acercamiento a las opiniones y actitudes de los estudiantes chinos hacia las herramientas de TAO y TA en la combinación chino-español. Para ello

Carmen VALERO-GARCÉS y Yanping TAN Análisis de las actitudes de estudiantes de traducción hacia las herramientas TAO...
CLINA

vol. 6-1, June 2020, 69-88

elSSN: 2444-1961

Ediciones Universidad de Salamanca - CC BY-NC-ND 
comenzaremos con un breve repaso de algunos estudios sobre diferentes combinaciones lingüísticas con el fin de conocer si ese avance de las tecnologías de la traducción en la formación de los futuros traductores está generalizado y si se da en los diferentes niveles de la formación. A continuación, mencionaremos algunos estudios que confirman ese interés a nivel global. Seguirá un breve recorrido sobre estudios que tratan de la traducción en relación con el chino para, en el siguiente paso, describir el estudio específicamente llevado a cabo en la combinación chino-español.

\section{ACTITUDES DE LOS ESTUDIANTES SOBRE LAS HERRAMIENTAS TAO}

Cuatro son los estudios a los que haremos referencia: los dos primeros se centran en la combinación español-inglés y los dos siguientes en otras distintas: árabe-inglés y turco-inglés. Si bien los estudios se realizaron en contextos distintos y con sujetos diferentes, nuestro propósito es comprobar en qué medida ofrecen datos sobre el interés de los estudiantes de traducción por las tecnologías de la traducción.

El primer estudio al que nos referiremos es de Olalla-Soler (2013), quien describe el conocimiento y uso de ciertas herramientas y recursos para la traducción de los estudiantes de Traducción e Interpretación de las universidades españolas a partir de los datos obtenidos en una encuesta. La muestra se compone de un total de 85 estudiantes de licenciatura y grado en Traducción e Interpretación de 18 universidades y 19 egresados. La encuesta pedía a los alumnos que indicasen, de todo un listado con herramientas de software libre o gratuitas y de pago, cuáles conocían, pero no usaban, cuáles desconocían y cuáles usaban frecuentemente.

En el caso de los alumnos en formación, la conclusión más relevante es que las herramientas de software libre o gratuitas (y, en concreto, los procesadores de texto y las herramientas TAO) suelen ser las más usadas. Una segunda conclusión indica que los altos valores registrados en las categorías «No lo conozco» y «Lo conozco, pero no lo uso» se dan en las herramientas de lingüística de corpus, gestión, alineación de textos y conversión de documentos, es decir, aquellas que no son centrales en el proceso traductor entendido como la primera acepción de Muñoz Martín $(2010,178)$.

En el caso de los egresados, se observan valores elevados en la categoría «Lo conozco y lo uso" en relación con listas de distribución, programas de lingüística de corpus, herramientas de gestión y alineadores de textos, aunque en ninguna herramienta se supera el 52,63 \%, que es el valor más alto registrado en la categoría «Lo conozco y lo uso» para los egresados.

En el segundo estudio que comentaremos, Sanz Villa (2015) lleva a cabo un análisis comparado de actitudes hacia el uso de herramientas TAO en cuatro grupos distintos. En el mismo participaron 234 personas, de las cuales 52 son traductores en formación y sin experiencia previa, 35 son traductores con menos de un año de experiencia,

Carmen VALERO-GARCÉS y Yanping TAN Análisis de las actitudes de estudiantes de traducción hacia las herramientas TAO...
CLINA

vol. 6-1, June 2020, 69-88

elSSN: 2444-1961

Ediciones Universidad de Salamanca - CC BY-NC-ND 
61 traductores con entre uno y cinco años de experiencia, y los 86 restantes son traductores con más de cinco años de experiencia. Las herramientas TAO analizadas se limitan a SDL Trados, Déjà Vu, Omega T, Wordfast, START Transit, Logoport y MemoQ. Teniendo en cuenta los objetivos de nuestro estudio, nos interesan los resultados registrados en los dos primeros grupos, es decir, el grupo de traductores en formación y sin experiencia previa, y el grupo de traductores con menos de un año de experiencia, que incluye a algunos que aún siguen formándose.

En cuanto al primer grupo de traductores en formación y sin experiencia previa, los datos indican que, entre las propuestas, la herramienta a la que se recurría con mayor frecuencia era SDL Trados, mientras que las que menos éxito cosechaban eran Logoport y STAR Transit. Se aprecia, sin embargo, una tendencia a utilizar más herramientas de software libre como OmegaT por cuestiones económicas y por la facilidad para conseguir el programa. La no utilización de herramientas TAO se atribuye al desconocimiento de los programas por parte de encuestados que aún estaban formándose y apenas habían adquirido nociones sobre ellas.

En cuanto al segundo grupo, el de traductores con menos de un año de experiencia, las respuestas sobre el empleo y frecuencia de uso de herramientas de traducción asistida por ordenador también difieren en cierta medida de las del grupo anterior: más del 50 \% de ellas elegían «sí, habitualmente» o «sí, siempre».

En cuanto a la herramienta más utilizada, de nuevo SDL Trados fue la que más puntuación obtuvo, aunque esta vez en mayor proporción (el 43 \% de los encuestados indicó que la utilizaba siempre). MemoQ experimenta valores más elevados respecto al perfil anterior. Estos datos concuerdan con los resultados del estudio de EUATC (2019) de 2019 en el que las empresas confirman el uso mayoritario de SDL Trados y un avance considerable de MemoQ y de Memsource, a la par que Déjà Vu y Wordfast experimentan cierto descenso.

Las personas que señalaron que no las utilizaban nunca aducían como razones que no sabían usarlas por falta de formación. Una de ellas mencionaba que le hacían perder más tiempo por basarse en la idea equivocada de que un segmento siempre se traduce de la misma manera.

Los trabajos de Mahfouz (2018) y de Çetiner (2018) (estudios 3 y 4) están centrados en entornos diferentes, alejados tanto lingüística como culturalmente, pero en ambos casos el inglés sigue siendo una de las lenguas de referencia.

El autor del tercer estudio (Mahfouz 2018) centra su interés en otra combinación lingüística en la que escasean las investigaciones sobre el tema que nos ocupa: inglés-árabe. La muestra analizada comprende a 114 estudiantes de traducción y traductores profesionales de Egipto. Mahfouz utiliza una metodología mixta que combina encuestas y entrevistas semiestructuradas. El estudio señala una actitud general favorable entre los participantes en relación con el uso de herramientas TAO. Los resultados también confirman que los usuarios con mejores conocimientos informáticos tienen actitudes más favorables hacia las herramientas TAO, en contraste con aquellos de los que tienen más experiencia en la traducción.

Carmen VALERO-GARCÉS y Yanping TAN Análisis de las actitudes de estudiantes de traducción hacia las herramientas TAO...
CLINA

vol. 6-1, June 2020, 69-88

eISSN: 2444-1961

Ediciones Universidad de Salamanca - CC BY-NC-ND 
Los datos de las entrevistas semiestructuradas revelan que, si bien la mayoría de los entrevistados están de acuerdo en que las ventajas básicas de las herramientas TAO son el ahorro de tiempo y el aumento de la productividad, muestran opiniones contradictorias sobre aspectos como su precio o el tiempo que se necesita para la actualización de las herramientas o para la revisión de los textos traducidos con dichas herramientas. Otro elemento de contradicción tiene que ver con la dependencia de la traducción automática. Ello es considerado por algunos como una buena oportunidad para sacar partido de la experiencia previa y por otros como un riesgo, dada la inexactitud de las traducciones. En general, casi todos los entrevistados coincidían en que las herramientas TAO no son asequibles.

El cuarto estudio que comentaremos es el de Çetiner (2018), quien trata de averiguar si hay un cambio en las actitudes de los estudiantes de traducción de la Universidad Kırıkkale (Turquía), después de tomar un curso sobre su uso. En él se utilizó Memsource Academic Edition como principal herramienta, junto con otros programas como Wordfast Anywhere, SDL Trados y Google Translators' Toolkit. El objetivo del curso era familiarizar a los estudiantes con las tecnologías de la traducción en general y con los principios de la TA. En el estudio participaron 66 estudiantes que tenían el turco como lengua materna y el inglés como lengua extranjera. El estudio tenía como objetivos principales investigar si se producía un cambio significativo en las actitudes de los estudiantes de traducción hacia las herramientas TAO después de tomar el curso y su opinión sobre la calidad de las traducciones realizadas con el apoyo de dichas herramientas.

Los resultados indican que los estudiantes desarrollaron una actitud positiva hacia la traducción asistida por ordenador después del curso. En cuanto a la calidad, se observa una actitud positiva. Este cambio puede deberse al hecho de que, al aprender a utilizar memorias de traducción, motores de búsqueda, bancos de términos, o corpus, los estudiantes se dieron cuenta de los beneficios prácticos del uso de herramientas de traducción de cara al control de calidad, en tanto supuso un aumento de la productividad al disminuir el tiempo empleado en la búsqueda de términos y al mejorar la eficiencia.

En definitiva, a pesar de lo limitado de los estudios presentados, podría afirmarse que el uso de herramientas TAO está integrado en la formación de futuros traductores en diversas combinaciones lingüísticas. Los estudiantes muestran actitudes positivas hacia ellas y diferentes grados de conocimiento en cuanto a su uso.

A continuación, nos centraremos en otros estudios relacionados con el chino como paso previo a la presentación del estudio realizado sobre el uso de las herramientas TAO en la combinación chino-español.

\section{ESTUDIOS SOBRE TECNOLOGÍA Y TRADUCCIÓN EN CHINA}

En 1985, se celebró la Primera Conferencia Nacional de Universidades de Lengua Extranjera sobre Lenguajes Audiovisuales en Pekín, que acogió un gran número de

Carmen VALERO-GARCÉS y Yanping TAN Análisis de las actitudes de estudiantes de traducción hacia las herramientas TAO...
CLINA

vol. 6-1, June 2020, 69-88

elSSN: 2444-1961

Ediciones Universidad de Salamanca - CC BY-NC-ND 
presentaciones sobre CALL (Aprendizaje de Idiomas Ayudado por Ordenador) y donde se mostró software de CALL. En 2002, la Universidad China de Hong Kong fue pionera en poner en marcha el Máster en Traducción Asistida por Ordenador (Chen 2012). En la China continental, la profesora Qian (2011) inició en 2004 el primer curso de traducción asistida por ordenador en la Universidad de Aeronáutica y Astronáutica de Beijing. Por su parte, en 2006, la Universidad de Pekín estableció el primer Máster sobre TAO en China y creó una serie de cursos relacionados con nuevas tecnologías de apoyo a la traducción y sus aplicaciones, aspectos terminológicos, proyectos de localización e internacionalización, gestión de proyectos de traducción, etc. (Zhang y Wang 2016).

A partir del año 2007, China empezó a establecer másteres en traducción e interpretación (MTI) en el país; 10 años después, hasta noviembre de 2016, 215 universidades chinas ofrecían dichos másteres.

En 2008, el Comité Directivo de Educación de MTI lanzó el Programa de Capacitación de Orientación de MTI, que incorpora cursos de «Traducción asistida por ordenador (TAO)» en el plan de estudios de MTI (Cui y Niu, 2016, 94). Sin embargo, hasta la fecha, la asignatura de TAO sigue siendo solamente optativa en el plan de estudios de MTI en China (Yao y Zhao 2017, 22).

Un informe publicado en 2008 sobre el uso de herramientas TAO en la industria de la traducción china mostró que alrededor del $66 \%$ de los traductores en China usaban estas herramientas: el 86 \% de los traductores empleaba SDL Trados, el 30 \% Yaxin, el 26 \% Déjà Vu y el $21 \%$ Wordfast (Zhu y Chen 2013, 70). En las universidades, la principal herramienta empleada en la docencia fue SDL Trados, que tiene la mayor cuota de mercado, mientras que el informe únicamente hace una breve alusión a Déjà Vu y Wordfast (Zhou 2013, 91).

En el plano de la investigación, según Yao y Zhao $(2017,22)$, entre 1988 y 2016 , se publicaron 631 artículos sobre herramientas TAO en cnki.net ${ }^{1}$, entre los cuales solo el 28,4 \% trataban cuestiones relativas a la enseñanza de TAO. Según estos autores, las principales razones de la escasez de investigaciones sobre la enseñanza de TAO en China se pueden resumir en tres. En primer lugar, TAO es una asignatura nueva en China. Fue en 2007 cuando la Universidad de Pekín ofreció el primer máster de TAO en el país; en 2013, solo 11 universidades chinas ofrecían asignaturas de TAO. Segundo, hay muy pocos docentes cualificados. Tercero, se observa una gran escasez de materiales didácticos al respecto. Además, hay que señalar que, debido a que el inglés es el idioma extranjero dominante que se aprende y utiliza en China, estas investigaciones también se basan en la combinación chino-inglés.

Si la investigación sobre la enseñanza de TAO en China es escasa, los estudios sobre las actitudes y opiniones de los estudiantes del MTI sobre TAO lo son todavía

1. CNKI es un proyecto a gran escala para publicar artículos científicos, disertaciones, anuarios y periódicos, entre otros, disponibles digitalmente a través de bases de datos. Se trata del mayor proveedor de recursos académicos en China.

Carmen VALERO-GARCÉS y Yanping TAN Análisis de las actitudes de estudiantes de traducción hacia las herramientas TAO...
CLINA

vol. 6-1, June 2020, 69-88

eISSN: 2444-1961

Ediciones Universidad de Salamanca - CC BY-NC-ND 
más, y prácticamente inexistentes para la combinación español-chino. A continuación, nos referiremos a algunos estudios sobre dicho tema en el par de lenguas chino-inglés.

Song, Zhang y Cheng (2011) realizaron una encuesta a 36 alumnos de la carrera Filología Inglesa de la Universidad de Economía y Derecho de Henan después de una formación de 8 semanas sobre TAO. Los resultados revelan que la motivación principal de la mayoría de los estudiantes para inscribirse en este curso era principalmente mejorar su nivel de traducción y aprobar el examen de esta materia. Muchos estudiantes no se habían dado cuenta de la importancia de los conocimientos y teorías asociadas a las herramientas de TAO (46).

Li $(2012,19)$ realizó una encuesta online a los estudiantes a nivel nacional que habían utilizado alguna herramienta TAO. Entre las 65 encuestas que recibió la autora, el $83 \%$ de los estudiantes mostraban una actitud positiva a estas herramientas y el $92 \%$ apoyaban la implementación de la enseñanza de las mismas. Sin embargo, el $16,92 \%$ de los participantes creían que las herramientas TAO eran solo un software pedagógico, y el 44,62 \% pensaban que se trataba de herramientas de software para prácticas de traducción. Además, el $71 \%$ de los alumnos estaban satisfechos con la formación de TAO que habían recibido, mientras que la mitad de los 65 encuestados sostenían que la enseñanza de las herramientas de TAO no tenía muchas diferencias respecto a la enseñanza de otros programas informáticos. Por otro lado, el 77 \% de los participantes afirmaban que no habían experimentado grandes dificultades para aprender a usar estas herramientas.

En cuanto a la metodología docente, el 44,62 \% de los estudiantes optaban por la combinación de explicación teórica y práctica; el 30,77 \% preferían el método de «aprendizaje por proyectos»; el 15,38 \%, votaban por el «aprendizaje por tareas»; y el 9,23\%, por la «enseñanza tradicional».

Liu (2017, 83-84) llevó a cabo un estudio sobre la actitud y opiniones acerca de las herramientas TAO de los 57 estudiantes de la carrera de filología inglesa de la Universidad Jimei de Fujian al término de una formación de 32 horas sobre el uso del SDL Trados 2014. Los resultados muestran que las herramientas que utilizaron en el proceso de traducción eran diversas. Ordenadas de mayor a menor, según su uso, emplearon: diccionarios online, SDL Trados 2014, traductores automáticos, corpus, diccionarios en dispositivos móviles, recursos online, entre otros. No obstante, solo el $56,14 \%$ de los alumnos estaban satisfechos con las herramientas TAO que habían utilizado; el 61,4 \% de los mismos pensaban que las traducciones automáticas que ofrecían no eran muy útiles o poco útiles; sin embargo, el 71,93 \% de los estudiantes afirmaban que la traducción automática podía mejorar la eficiencia traductora. Por otro lado, a pesar de que la mayor dificultad que encontraban los alumnos era la gestión de los términos, sobre todo los términos especializados, la utilización de bases de datos terminológicas no era alta: el 73,68 \% de ellos afirmaban que no las habían utilizado o las utilizaron poco, mientras que el $68,42 \%$ eran optimistas sobre su utilidad y las calificaban de útiles o muy útiles.

Carmen VALERO-GARCÉS y Yanping TAN Análisis de las actitudes de estudiantes de traducción hacia las herramientas TAO...
CLINA

vol. 6-1, June 2020, 69-88

elSSN: 2444-1961

Ediciones Universidad de Salamanca - CC BY-NC-ND 
Estos datos muestran que los estudiantes tenían altas expectativas sobre el uso del software de TAO y eran positivos sobre el futuro de este tipo de software, pero no estaban satisfechos con la efectividad real de los programas.

Ge (2018), en su estudio sobre la situación de la enseñanza de TAO en universidades de Shanghai, señala que la mayoría de los estudiantes tienen una actitud positiva sobre el uso de dichas herramientas: el $68,75 \%$ creen que el dominio de las mismas resulta importante para su desarrollo profesional; el 77,84 \% creen que hay que ampliar la duración de la enseñanza sobre estas herramientas, y solo un $6 \%$ de los alumnos las usan con frecuencia. De hecho, casi un tercio de los encuestados piensan que sería mejor aprender a usarlas después de graduarse, una vez se enfrenten a problemas prácticos en su trabajo, ya que en esa situación estarían más motivados y el efecto de aprendizaje sería mayor. El autor también realizó entrevistas en algunas empresas de traducción, las cuales declaraban hacer uso de las herramientas TAO en los encargos de traducción escrita; sin embargo, la encuesta revelaba que la mayoría de los traductores no recibían formación al respecto tras entrar en la empresa, por lo que se espera que los estudiantes de MIT dominen dichas herramientas al término de su formación universitaria y se reduzca así el coste de formación proporcionada por las empresas (29-30).

En la pregunta sobre las deficiencias del software, los estudiantes identificaron las siguientes deficiencias en SDL Trados 2014: coste elevado, difícil manejo y la calidad poco satisfactoria, dado que hay fallos de precisión en la gestión del vocabulario, errores gramaticales y de sintaxis. No obstante, los estudiantes también señalaron ventajas del software: es rápido, conveniente y permite ahorrar mucho tiempo.

\section{ESTUDIO DE CASO SOBRE LA ACTITUD DE ESTUDIANTES CHINOS HACIA EL USO DE LA TECNOLOGÍA EN TRADUCCIÓN (CHINO-ESPAÑOL)}

\subsection{Metodología}

La metodología utilizada combina métodos cuantitativos y cualitativos. Los datos cuantitativos proceden de una encuesta sobre las actitudes hacia las herramientas TAO realizada entre los estudiantes del MA en Comunicación Intercultural, Interpretación y Traducción en los Servicios Públicos² (MA en CITISP) de la UAH de la combinación chino-español durante el curso 2018-2019 y 2019-2020 basándonos en los estudios previos mencionados (Olalla-Soler 2013; Sanz Villa 2015; Mahfouz 2018 y Çetiner 2018). Los datos cualitativos provienen de la observación como formadores y tutores de los sujetos objeto de estudio sobre el programa de estudios, el componente tecnológico y los recursos disponibles del MA, así como sobre su percepción acerca

\section{Para más información sobre el máster, véase: http://www3.uah.es/master-tisp-uah/}

Carmen VALERO-GARCÉS y Yanping TAN Análisis de las actitudes de estudiantes de traducción hacia las herramientas TAO...
CLINA

vol. 6-1, June 2020, 69-88

elSSN: 2444-1961

Ediciones Universidad de Salamanca - CC BY-NC-ND 
de la aplicabilidad de las tecnologías de traducción y sus sugerencias para la reforma del plan de estudios.

Con este fin se elaboró un cuestionario que completaron los alumnos de la especialidad chino-español. El número total de participantes fue 60.

MA CITISP tiene una carga lectiva de 60 ECTS y contiene asignaturas susceptibles de usar herramientas TAO, tales como «Técnicas y Recursos para la Traducción e Interpretación en los Servicios Públicos» (6 ECTS) y otras relacionadas con la interpretación y traducción en el ámbito sanitario o en el ámbito administrativo y jurídico. En el caso de chino, los alumnos recibieron a lo largo del Máster un total de 24 horas (3 ECTS) en la asignatura de «Técnicas y Recursos», entre las cuales 1 ECTS se dedicó a terminología y otros dos a una introducción a las herramientas TAO.

El cuestionario se repartió a los alumnos al término de estas sesiones, y constaba de 5 partes principales: cuestiones generales, recursos y herramientas, traducción asistida por ordenador (TAO), traducción automática (TA) y terminología. Al final de la encuesta, se formulaban dos preguntas adicionales para saber su satisfacción sobre esta formación tecnológica específica.

A continuación, se analizan los resultados de las encuestas por secciones.

\subsection{Análisis de resultados}

\subsubsection{Cuestiones generales}

En esta parte se pretende conocer los perfiles de los encuestados. Todos son estudiantes del máster arriba mencionado, su lengua materna es el chino, todos dominan el español a nivel intermedio o avanzado, y el tiempo de residencia en España varía entre algunos meses y varios años.

\subsubsection{Recursos y herramientas}

En los recursos y herramientas, se pregunta sobre el uso de herramientas TAO como procesadores de texto, conversores de documentos, alineadores de textos, gestores de correo, buscadores generales, buscadores especializados, herramientas de gestión, programas de lingüística de corpus y listas de distribución. Los alumnos tenían que elegir entre las opciones: 1. Uso frecuente; 2. Uso alguna vez; 3. Lo conozco, pero no lo uso; 4. No lo conozco.

El análisis de los resultados muestra lo siguiente: un 53 \% de los alumnos usan Google Drive (gratuito) frecuentemente; un $77 \%$ de los mismos usan Microsoft Word y Open Office (libre y gratuito) con frecuencia; sin embargo, la mayoría no conocen Libre Office (libre y gratuito).

Carmen VALERO-GARCÉS y Yanping TAN Análisis de las actitudes de estudiantes de traducción hacia las herramientas TAO... 
En cuanto a las herramientas TAO, entre las opciones que se facilitaban, CatsCradle, Déjà Vu, MemoQ, OmegaT (libre y gratuito), SDLX, Trados, Transit y Wordfast, solo un $28 \%$ de los alumnos usan Wordfast frecuentemente, mientras que el resto (es decir, la absoluta mayoría de los alumnos) o bien las conoce, pero no las usa, o bien no las conoce. Esto incluye SDL Trados. Pasa lo mismo con los conversores de documentos y los alineadores de textos, ya que la mayoría de los alumnos no las conocen, exceptuando WinAlign, que es utilizado alguna vez por un $35 \%$ de los alumnos.

En cuanto a los gestores de correo, la mayoría usan Gmail frecuentemente (63\% de los entrevistados) y un $33 \%$ usan Outlook con frecuencia, mientras que casi nadie conoce Thunderbird.

Sobre los buscadores, GoogleSearch prevalece sobre los demás, ya que un $97 \%$ de los alumnos lo usan frecuentemente, mientras que casi nadie usa a menudo Bing o Yahoo. En cuanto a los buscadores especializados, solo un $42 \%$ usan GoogleScholar con frecuencia, mientras que la mayoría no conocen Scirus.

En cuanto a las herramientas de gestión, los programas de lingüística de corpus y las listas de distribución, la absoluta mayoría de los alumnos de los dos grupos no conoce ninguna de las herramientas listadas.

La conclusión que se puede sacar es que los entrevistados tienen escasos conocimientos acerca de este tipo de recursos y herramientas.

\subsubsection{Traducción asistida por ordenador (TAO)}

Esta parte intenta, a través de las 9 preguntas siguientes, conocer los conocimientos y actitudes de los alumnos:

1. ¿Conoce herramientas de Traducción Asistida por Ordenador (TAO) (como p. ej. SDL Trados, Déjà Vu, etc.)?

2. En caso afirmativo, ¿cuál/es de las siguientes herramientas conoce? Marque solo la que haya utilizado al menos una vez.

3. ¿Conoce otras herramientas TAO de uso en su combinación lingüística?

4. Valore de 1 a 5 su dominio de las herramientas TAO. 1: inexperto y 5: experto.

5. ¿Cómo ha adquirido los conocimientos de TAO?

6. ¿Considera que para tener éxito profesional necesita mejorar sus conocimientos actuales de TAO?

7. Señale de 1 a 5 su grado de conformidad con la siguiente afirmación: «Todo traductor necesita poseer al menos un dominio básico de TAO para competir en el mercado». 1: en total desacuerdo y 5: completamente de acuerdo.

8. ¿Para cuál/es de los siguientes elementos cree que es más productivo traducir con herramientas TAO que sin ellas? Marque todas las que estime oportuno.

9. ¿Para los textos de qué ámbito resultan más útiles las TAO? Marque solo el ámbito de los textos en los que utilice estas herramientas.

Carmen VALERO-GARCÉS y Yanping TAN Análisis de las actitudes de estudiantes de traducción hacia las herramientas TAO...
CLINA

vol. 6-1, June 2020, 69-88

elSSN: 2444-1961

Ediciones Universidad de Salamanca - CC BY-NC-ND 
El análisis de los resultados muestra lo siguiente:

Preguntas 1 y 2: el $75 \%$ de los alumnos admiten que las conocen, pero no las usan o las usan ocasionalmente; solo el $23 \%$ de ellos las usan a menudo. Entre las 4 herramientas listadas (SDL Trados, Déjà Vu, OmegaT y Wordfast), según el total de los alumnos encuestados que las han usado al menos una vez, Wordfast resulta ser la más utilizada, ya que un $97 \%$ la han probado; la que ocupa el segundo lugar es OmegaT, ya que un $43 \%$ de los estudiantes la han utilizado por lo menos una vez; el porcentaje tanto para SDL Trados como para Déjà Vu resulta ser el mismo, un 12 \% (Gráfico 1) .

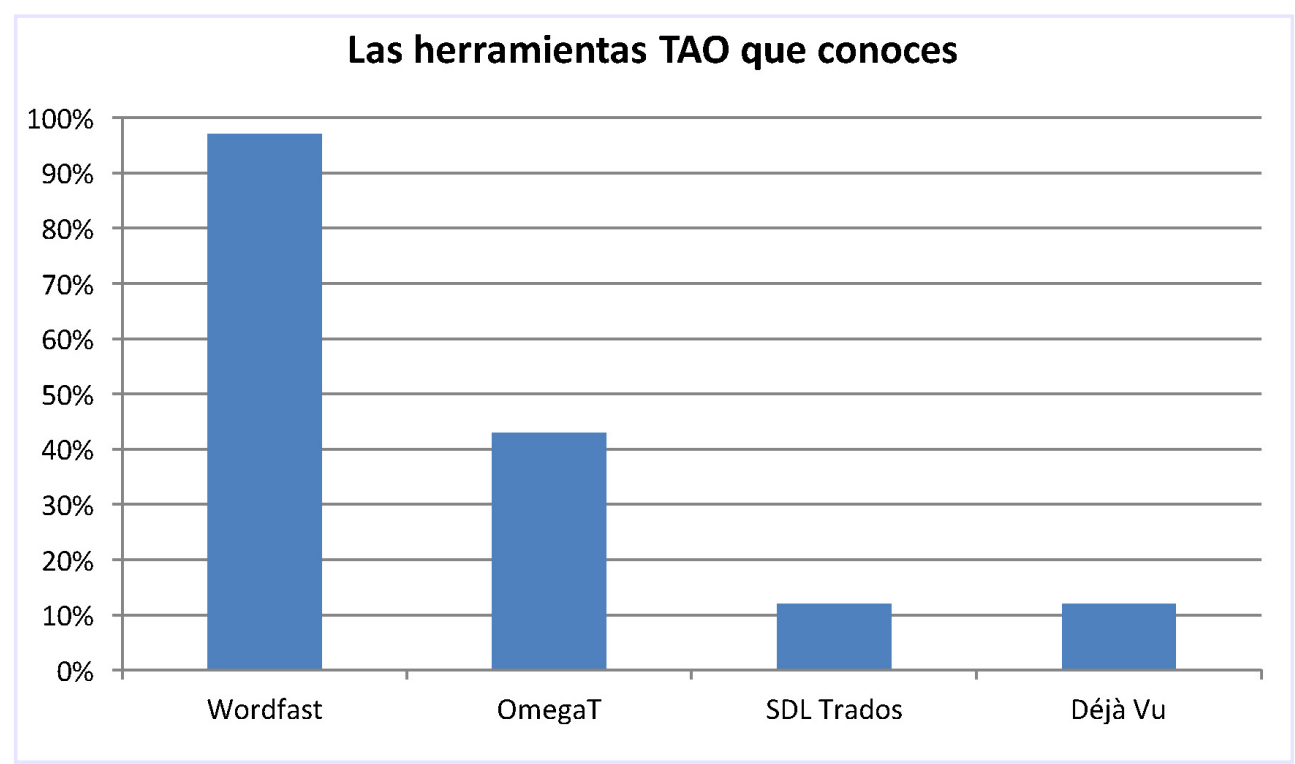

Gráfico 1. Las herramientas TAO que conoces

El resultado de la pregunta 3, acerca de si conocen otras herramientas TAO de uso en su combinación lingüística, demuestra que un $47 \%$ de los alumnos no las conocen; un $25 \%$ afirman que las conocen, pero no las usan; y solo el $5 \%$ indican que las usan a menudo. Estos resultados revelan que la mayoría de los alumnos chinos no utilizan ninguna herramienta TAO para su combinación lingüística de trabajo. En cuanto a su dominio de las mismas, ninguno considera que sea experto (nivel puntuado con un 5), un $8 \%$ creen que su dominio se corresponde con un 4, un $5 \%$ piensan que son inexpertos (un 1), mientras que un $83 \%$ se deciden por el 2 y el 3 .

Sobre cómo han adquirido los conocimientos de TAO, el $95 \%$ de los estudiantes indican que los han adquirido en la universidad; esta cifra confirma que las universidades siguen siendo la principal fuente de la enseñanza y vía de formación en las herramientas de TAO.

Carmen VALERO-GARCÉS y Yanping TAN Análisis de las actitudes de estudiantes de traducción hacia las herramientas TAO... 
El resultado de la siguiente pregunta, sobre si consideran que para tener éxito profesional necesitan mejorar sus conocimientos actuales de TAO, revela que un $88 \%$ de los alumnos consideran que necesitan mejorar en parte o en gran medida sus conocimientos actuales de TAO para tener éxito profesional, mientras que solo el $8 \%$ declaran que no saben usarlas, pero tampoco lo consideran necesario. Estas cifras exponen la actitud positiva de los alumnos hacia la formación y el uso de las herramientas TAO.

El resultado de la siguiente pregunta reafirma esa misma idea, ya que el $92 \%$ de los alumnos están de acuerdo con que «todo traductor necesita poseer al menos un dominio básico de TAO para competir en el mercado». Combinando el resultado de estas últimas dos preguntas, junto con el de la anterior, según la cual el $95 \%$ de los alumnos han adquirido sus conocimientos de TAO en la universidad, parece claro que esta última debería tenerlo en cuenta a la hora de diseñar el plan de estudios.

En cuanto a la pregunta sobre los elementos por los que considera que es más productivo traducir con herramientas TAO que sin ellas, los datos indican lo siguiente:

\section{¿Para cuál/es de los siguientes elementos cree que es más productivo traducir con herramientas TAO que sin ellas?}

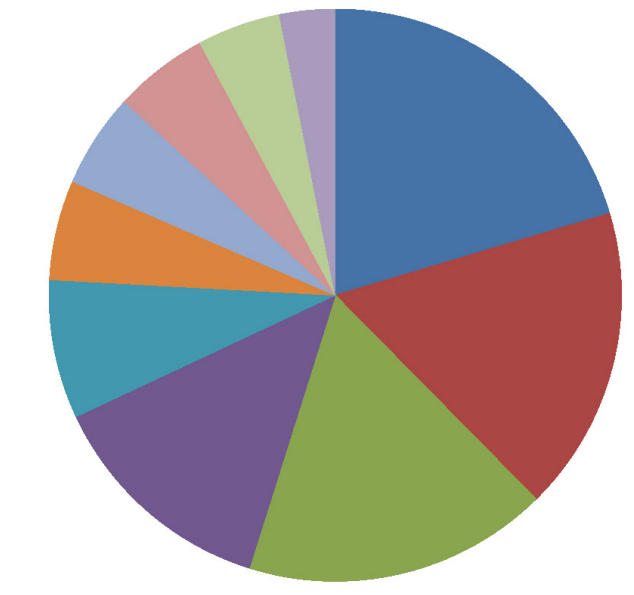

- Rapidez en la ejecución (65\%)

- Transferencia de los datos (55\%)

- Coherencia terminológica (55\%)

Formato (cursiva, negrita) (42\%)

- Ortografía (25\%)

- Fluidez del texto meta (18\%)

Puntuación (17\%)

Tablas (17\%)

$\square$ Sintaxis $(15 \%)$

Gráfico 2. Elementos en los que el uso de herramientas TAO resulta productivo

Los cuatro aspectos más importantes que destacan los estudiantes del uso de herramientas TAO son la rapidez en la ejecución, en la transferencia de los datos, en la coherencia terminológica y el formato (cursiva, negrita); los otros listados como la ortografía, la fluidez del texto meta, la puntuación, las tablas, la sintaxis y las referencias culturales resultan ser menos relevantes.

En cuanto a la pregunta sobre los tipos de texto para los que las herramientas TAO resultan más útiles, los datos indican lo siguiente (Gráfico 3):

Carmen VALERO-GARCÉS y Yanping TAN Análisis de las actitudes de estudiantes de traducción hacia las herramientas TAO... 


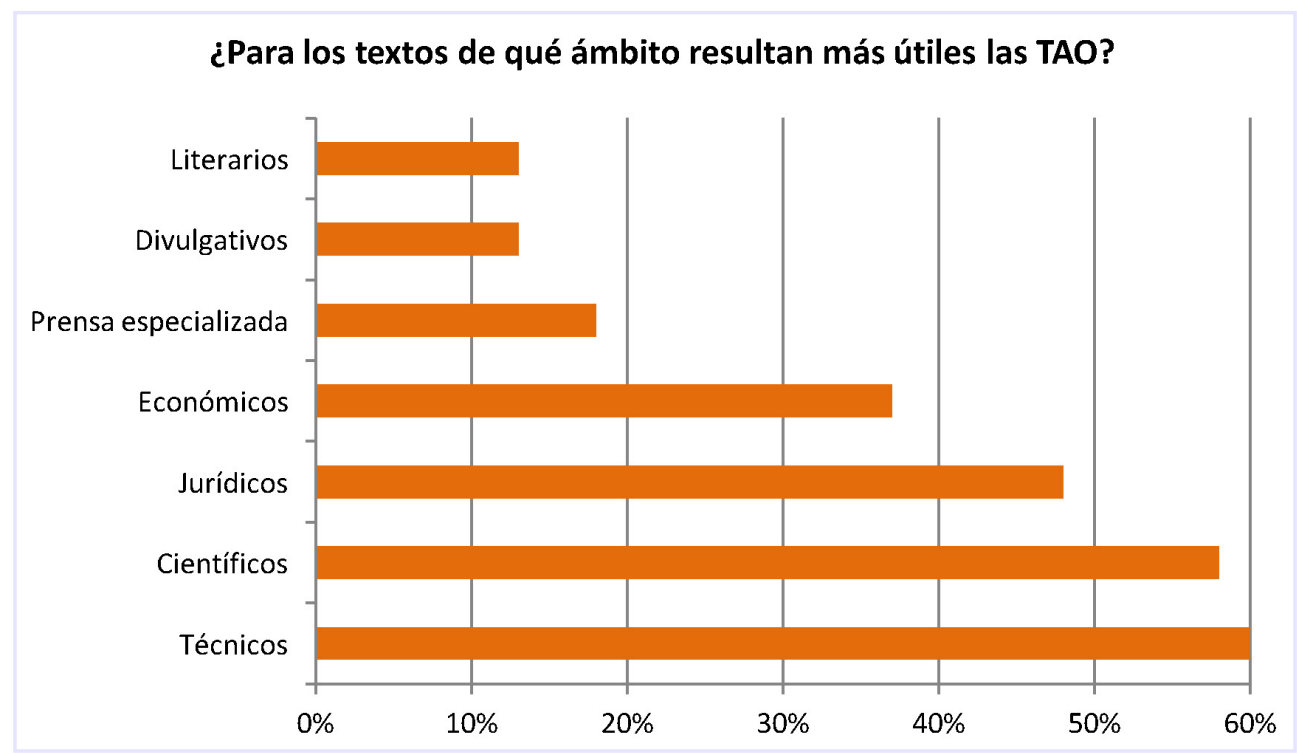

Gráfico 3. Para los ámbitos más útiles las TAO

Como bien demuestra el gráfico 3, los textos para los que los estudiantes creen que resultan más útiles las TAO, ordenados de mayor a menor, son los pertenecientes a los siguientes ámbitos: los técnicos, los cientíicos, los jurídicos, los económicos, la prensa especializada, los divulgados y los literarios.

\subsubsection{Traducción Automática (TA)}

Con respecto a la TA, los encuestados tienen que contestar a 7 preguntas de opción múltiple.

1. ¿Se apoya en la traducción automática (TA) para traducir?

2. En caso afirmativo, ¿por qué razones traduce con TA? Seleccione todos los que correspondan.

3. ¿Qué experiencia tiene en el uso de traducción automática TA + post-edición? (La post-edición se refiere a la corrección de un texto traducido de forma automática con el fin de que adquiera un resultado de calidad para su divulgación).

4. En el caso de utilizar memorias de traducción, ¿utiliza la opción de TA integrada en su herramienta TAO cuando la memoria no ofrece coincidencias?

5. Indique su grado de conformidad con la siguiente afirmación: «La TA ayuda a aumentar la productividad»: 1: en total desacuerdo y 5: completamente de acuerdo.

Carmen VALERO-GARCÉS y Yanping TAN Análisis de las actitudes de estudiantes de traducción hacia las herramientas TAO... 
6. Imagine que está trabajando para una agencia, si el cliente NO le pide expresamente que traduzca con TA, ¿cree que debe informarle si utiliza esta técnica en un encargo?

7. ¿En qué medida considera que el uso de la TA/post-edición reemplazará a la traducción tradicional? 1: Nada probable y 5: completamente probable.

Los resultados indican lo siguiente: sobre la primera pregunta, si los alumnos que se apoyan en la traducción automática (TA) para traducir, un $37 \%$ de ellos afirman que lo hacen «habitualmente»; el $40 \%$, alguna vez; solo un $7 \%$ enuncian que la usan siempre. El resto de los alumnos, o no la utiliza nunca, o raras veces. En cuanto a las razones por las que traducen con TA, el $55 \%$ confirman que les ayuda a comprender el texto; un $70 \%$ indican que traducen más rápido con TA. Las demás opciones (como «porque lo requiere mi empresa o institución», «porque lo demanda el cliente» o "porque yo lo prefiero") han sido seleccionadas por muy pocos alumnos. Observamos unas contradicciones entre las respuestas de estas dos preguntas, ya que bastantes estudiantes confirman que la TA les ayuda o bien a comprender el texto o bien a traducir más rápido, pero el porcentaje que declara el uso habitual de la TA resulta muy bajo: solo un $37 \%$.

En cuanto a la experiencia que tienen los alumnos en el uso de la TA + post-edición, entre los dos grupos, el $8 \%$ afirma que no tiene ninguna, el 27 \% que únicamente una experiencia escasa, el $30 \%$ intermedia, el $27 \%$ bastante y el $8 \%$ mucha. La siguiente pregunta, que inquiere si en el caso de utilizar memorias de traducción utilizan la opción de TA integrada en su herramienta TAO cuando la memoria no ofrece coincidencias, el $18 \%$ responden «nunca», el $38 \%$ "rara vez", el $40 \%$ "alguna vez", y solo un $2 \%$ señala que la usa «habitualmente» $y$ «siempre», respectivamente. Estos datos revelan que tanto el uso de la TA + post-edición como la integración de la TA en las herramientas TAO de los alumnos cuyos idiomas de trabajo son el español y el chino resultan bastante escasos; dicho de otro modo, todavía no tienen la costumbre de usar estas tecnologías.

Sin embargo, en relación con la pregunta sobre si están de acuerdo con que la TA ayuda a aumentar la productividad, el $17 \%$ afirman estar totalmente de acuerdo (el grado 5), el $37 \%$ muy de acuerdo (el 4) y el $33 \%$ de acuerdo (el 3); los que han elegido el 2 y el 1 (en total desacuerdo) solo suponen un $5 \%$ y un $7 \%$, respectivamente. Es decir, un $87 \%$ de los alumnos sí creen que la TA ayuda a aumentar la productividad, a pesar de que datos anteriores señalan que solo el $35 \%$ de los alumnos usan la TA + post-edición con frecuencia, lo cual debería llevar a reflexiones sobre las posibles razones (Gráfico 4).

Carmen VALERO-GARCÉS y Yanping TAN Análisis de las actitudes de estudiantes de traducción hacia las herramientas TAO...
CLINA

vol. 6-1, June 2020, 69-88

elSSN: 2444-1961

Ediciones Universidad de Salamanca - CC BY-NC-ND 


\section{Indique su grado de conformidad con la siguiente afirmación: "La TA ayuda a aumentar la productividad": 1: en total desacuerdo; 5: completamente de acuerdo}

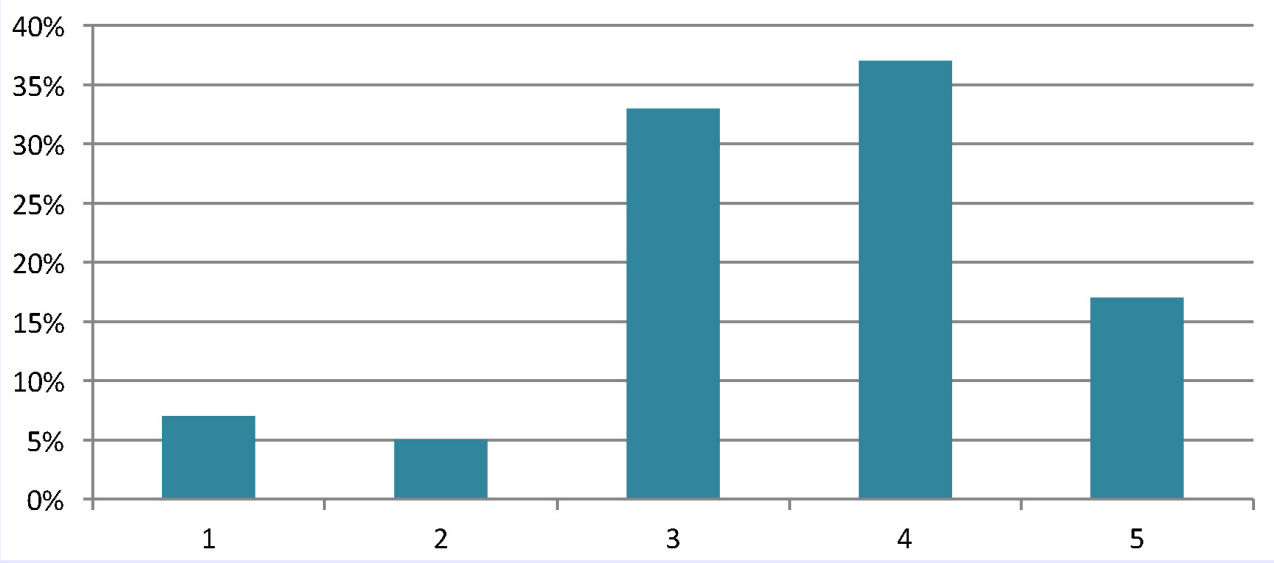

Gráfico 4. ¿La TA ayuda a aumentar la productividad?

La siguiente pregunta va más encaminada a conocer su opinión sobre un tema de la vida profesional. Ante la pregunta de si cree que debe informar de si utiliza la TA en un encargo en el que no se requiere expresamente, el $52 \%$ de los entrevistados manifiestan que sí, y el $48 \%$, que no. Dado que esta pregunta todavía no tiene una respuesta de consenso en el ámbito de la traducción, las respuestas de los alumnos también se hacen eco de esta realidad.

La última pregunta de esta sección iba dirigida a conocer en qué medida consideraba el alumno que el uso de la TA/post-edición reemplazará a la traducción tradicional. Se pedía que indicasen el porcentaje entre 1 y 5: 1: Nada probable y 5: completamente probable.

Los resultados son los siguientes: el $24 \%$ de los alumnos eligen nada probable, el $21 \%$ poco probable, el $40 \%$ entre nada probable y completamente probable (el 3); los que responden con el 4 y 5 solo suman un $16 \%$. Estos datos demuestran que la mayoría de los estudiantes aún no tienen mucha fe en la TA/post-edición. Si comparamos este resultado con el de que un $87 \%$ de los alumnos sí creen que la TA ayuda a aumentar la productividad, podríamos sacar la conclusión de que los entrevistados piensan que la TA mejora la productividad de la traducción, pero no reemplazará a la traducción tradicional; además, sería interesante contrastar esta actitud con la de los alumnos de otras lenguas (Gráfico 5).

Carmen VALERO-GARCÉS y Yanping TAN Análisis de las actitudes de estudiantes de traducción hacia las herramientas TAO... 


\section{¿En qué medida considera que el uso de la TA/post-edición reemplazará a la traducción tradicional? \\ 1: nada probable; 5: completamente probable}

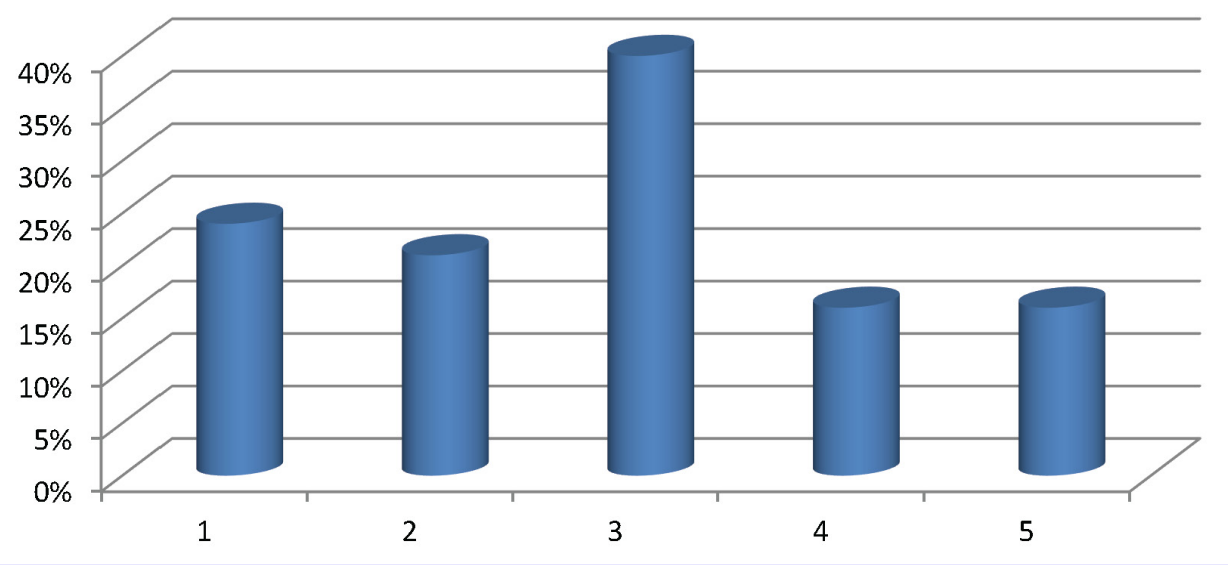

Gráfico 5. ¿La TA/post-edición reemplazará a la traducción tradicional?

\subsubsection{Terminología}

En esta parte se plantean 2 preguntas optativas sobre la terminología:

1) De las siguientes opciones, ¿cuál es la que más utiliza para gestionar terminología?

- Hojas de cálculo de Excel

- Bases de datos de Access

- Gestores terminológicos como Multiterm

- No almaceno terminología

- Otro:

2) Señale su grado de conformidad con la siguiente afirmación: «Las TAO facilitan la búsqueda y recuperación de la terminología». 1: en total desacuerdo y 5: completamente de acuerdo.

Las respuestas de los alumnos a la primera pregunta muestran que la absoluta mayoría de los alumnos únicamente usan Word (un 60 \%) y Excel (un 66 \%) para gestionar la terminología, mientras que los que utilizan Access y gestores terminológicos como Multiterm constituyen solo un $5 \%$ y $2 \%$, respectivamente; además, un encuestado (lo que supone el 2 \%) afirma que no almacena la terminología; en ningún caso se utilizan otras herramientas. El resultado de esta pregunta revela que Word y Excel siguen siendo las herramientas más utilizadas para gestionar la terminología por los

Carmen VALERO-GARCÉS y Yanping TAN Análisis de las actitudes de estudiantes de traducción hacia las herramientas TAO... 
encuestados; de hecho, parece que ya no conocen prácticamente otras herramientas destinadas a tal uso.

En cuanto a la segunda pregunta, nadie está en total desacuerdo, y los que han elegido el 2, 3, 4 y 5 ocupan, respectivamente, un $10 \%$, un $32 \%$, un $44 \%$ y un $14 \%$; es decir, la mayoría de los alumnos están de acuerdo, aun en diferente grado, en que las TAO facilitan la búsqueda y recuperación de la terminología.

Al final del cuestionario, se añaden dos preguntas para conocer las opiniones de los alumnos sobre la formación que han recibido en el Máster.

La primera inquiere si consideran útil la información que se da en el máster de CI\&ITSP sobre herramientas TAO y TA, del 1 al 5 (1: No, 5: Sî). El porcentaje, del 1 al 5 , resulta ser, respectivamente, $10 \%, 14 \%, 17 \%, 32 \%$ y $27 \%$. Estas cifras demuestran que solo un $24 \%$ de los estudiantes creen que la información no es útil, mientras que la mayoría (un 76 \%) opinan que sí les parece valiosa (Gráfico 6).

\section{¿Es útil la información que se da en el máster de CI\&ITSP sobre herramientas TAO y TA? \\ Del 1 al 5 (1: No, 5: Sí)}

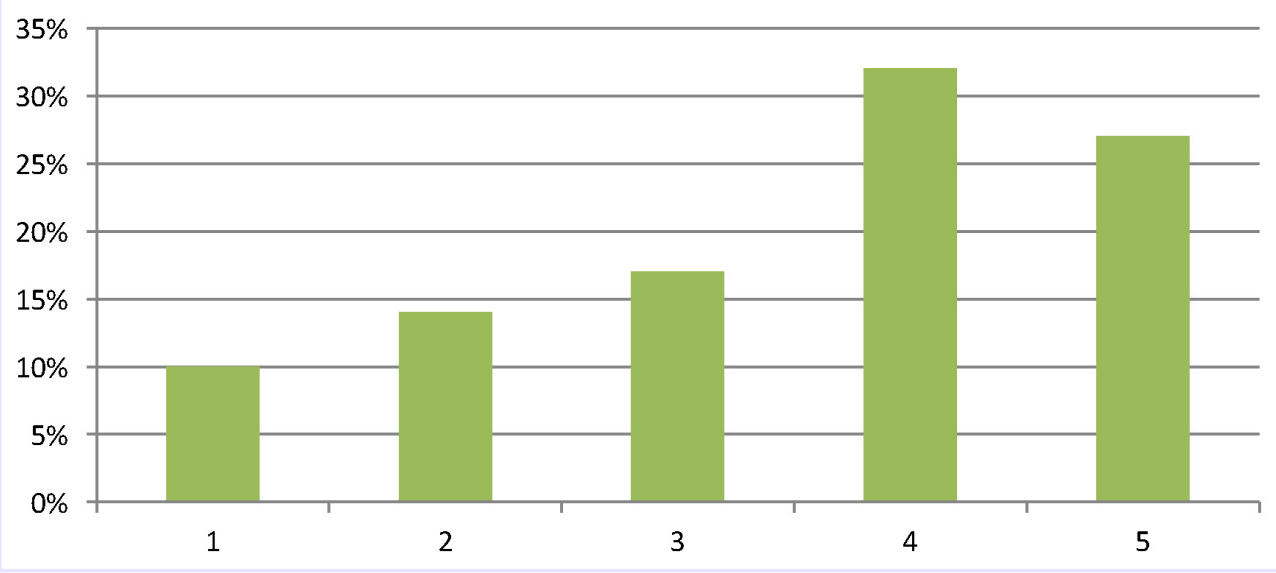

Gráfico 6. ¿Es útil la información que se da en el máster sobre herramientas TAO y TA?

La segunda pregunta trata de averiguar si creen que es suficiente dicha formación. Un 19 \% de los alumnos piensan que dicha formación no es suficiente, 44 \% creen que es adecuada y $37 \%$ sostiene una postura media (Gráfico 7).

Estas respuestas merecen una reflexión más profunda, ya que, como comentábamos al principio, los alumnos recibieron una formación introductoria de 24 horas en total sobre terminología y traducción asistida (TAO) antes de hacer la encuesta. Como es sabido, el uso de las herramientas TAO no es sencillo. Los datos muestran que 24

Carmen VALERO-GARCÉS y Yanping TAN Análisis de las actitudes de estudiantes de traducción hacia las herramientas TAO... 
horas de formación es claramente insuficiente, sobre todo para los alumnos que no habían recibido dicha formación con anterioridad (Gráfico 7).

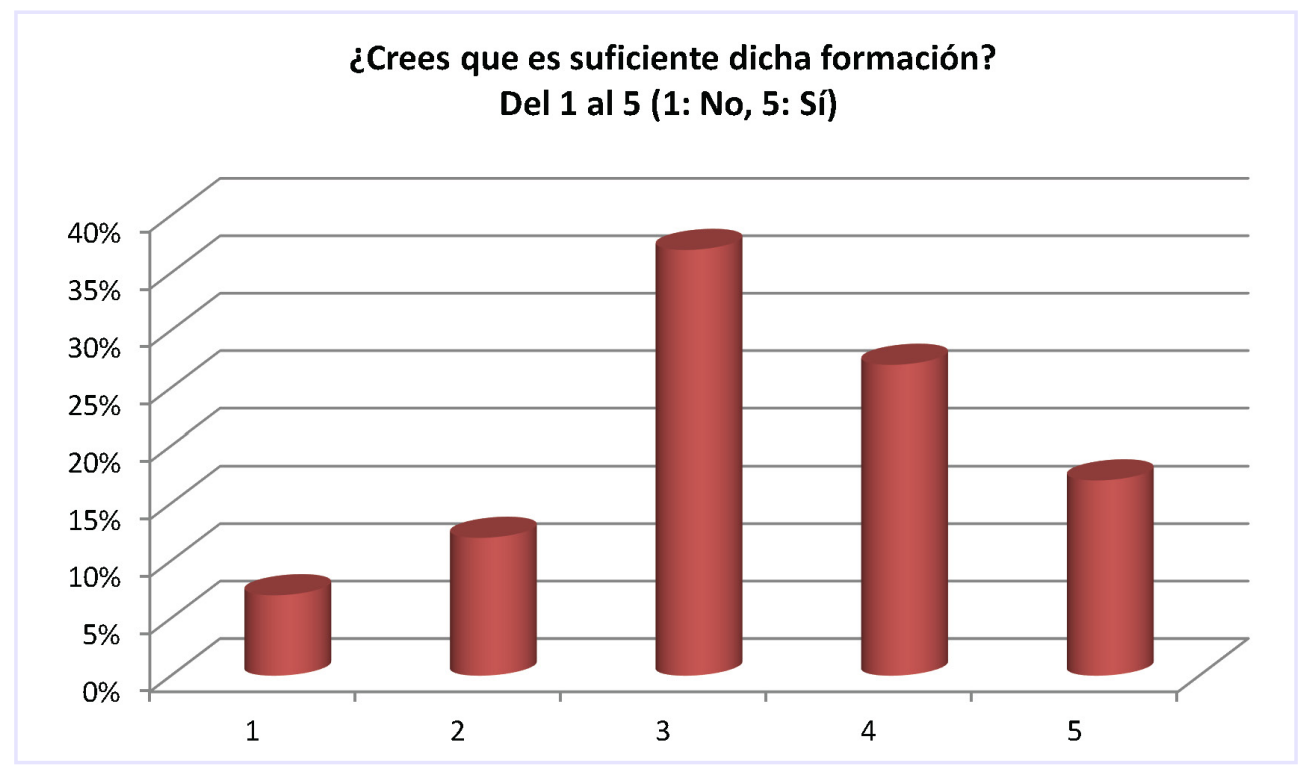

Gráfico 7. ¿Crees que es suficiente dicha formación?

En este sentido, los alumnos opinan que estas herramientas TAO y la formación que ofrece el máster son útiles, si bien los resultados de la primera parte de la encuesta revelan que tienen escasos conocimientos sobre las mismas, por lo que en teoría deberían pedir más formación al respecto; sin embargo, según los datos de esta pregunta, solo el $19 \%$ de ellos piensan que la formación que han recibido no es suficiente, frente al $44 \%$ que cree que es adecuada y el $37 \%$ que mantiene una postura media. Sería adecuado realizar una entrevista a los encuestados para conocer las razones de sus respuestas, ya que, a pesar de que se les pedía que razonaran su respuesta, los alumnos no ofrecieron comentario alguno.

\section{CONCLUSIONES}

Con el objetivo de conocer las actitudes de los estudiantes de traducción hacia las tecnologías de traducción, se ha llevado a cabo un breve análisis de diferentes estudios sobre el tema en diferentes combinaciones lingüísticas y contextos culturales y niveles de formación. En todos ellos se indica una actitud positiva por parte del alumnado y de los traductores profesionales, si bien se apuntan también algunas desventajas. En el caso del chino-español, tras un breve repaso por estudios relacionados con el objetivo

Carmen VALERO-GARCÉS y Yanping TAN Análisis de las actitudes de estudiantes de traducción hacia las herramientas TAO... 
del artículo, se ha realizado un estudio de caso como un primer acercamiento a las opiniones y disposiciones de los estudiantes chinos de traducción e interpretación sobre las herramientas de TAO y TA en la combinación chino-español. Tanto los estudios como los resultados de nuestro trabajo coinciden con las observaciones de otros investigadores como Christensen y Schjoldager (2010), que afirman que hay un consenso generalizado sobre el hecho de que la tecnología TAO está aquí para quedarse.

Como comentábamos anteriormente, un $92 \%$ de nuestros entrevistados están de acuerdo con que «todo traductor necesita poseer al menos un dominio básico de TAO para competir en el mercado" y un $87 \%$ de los alumnos creen que la TA ayuda a aumentar la productividad. Como bien apunta Krüger $(2016,114)$, los buenos tiempos de la traducción a pluma y en papel están inevitablemente llegando a su fin. La cuestión que se plantea ahora a los traductores y a esos futuros traductores objeto de nuestro estudio no es solo si utilizar o no herramientas informáticas, sino más bien qué herramientas comprar, aprender y utilizar, dada la gran variedad de recursos existentes, que crecen día a día.

Otra de las conclusiones derivadas del estudio sugiere que estamos ante un punto de inflexión, pues se está produciendo una proliferación de herramientas TAO y avances en la TA que, sin duda, van a afectar o están afectando ya a la imagen del traductor y de la profesión de la traducción en su conjunto y, en consecuencia, a la forma de preparar a los futuros traductores para el mercado. De acuerdo con el resultado de nuestra encuesta, la mayoría de los alumnos chinos no utilizan ninguna herramienta TAO para su combinación lingüística de trabajo, y el $75 \%$ de ellos admiten que las conocen, pero no las usan o las usan ocasionalmente, por lo que un $88 \%$ de los estudiantes consideran que necesitan mejorar en parte o en gran medida sus conocimientos actuales de TAO para tener éxito profesional.

Por último, los resultados de nuestra investigación también revelan que el 95 \% de los estudiantes han adquirido los conocimientos de TAO en la universidad, cifra que merece una reflexión más profunda tanto por parte de los docentes de traducción, en lo relativo al diseño curricular y la inclusión en el mismo de asignaturas o un porcentaje mayor de contenidos relativos a las tecnologías aplicadas a la traducción, como por parte de las empresas de traducción o proveedores de servicios lingüísticos, que deberán tener en cuenta el uso de herramientas TAO en su estrategia de venta o en la promoción de sus productos, pero también en la formación de sus propios empleados.

\section{BIBLIOGRAFÍA}

ÇETINER, Caner. 2018. «Analyzing the Attitudes of Translation Students towards CAT (Computer-Aided Translation) Tools». Journal of Language and Linguistic Studies, 14 (1), 153-161.

CHEN, Shanwei. 2012. «Translation Technology: Past, Present and Future». Conferencia pronunciada en la 2012 LTTC International Conference The making of a translator celebrada en Taipei.

Christensen, Tina Paulsen y Anne SChJoldager (2010). «Translation-Memory (TM) Research: What

Do We Know and How Do We Know It? ». Hermes, 44: 89-101.

Carmen VALERO-GARCÉS y Yanping TAN Análisis de las actitudes de estudiantes de traducción hacia las herramientas TAO...
CLINA

vol. 6-1, June 2020, 69-88

elSSN: 2444-1961

Ediciones Universidad de Salamanca - CC BY-NC-ND 
CUI, Qiliang y Niu SHUO. 2016. 《翻译专业硕士的计算机辅助翻译课程教学-基于ISO17100的翻 译技术分类标准 [Enseñanza de la traducción asistida por ordenador en los MTI- estándar de clasificación para la tecnología de traducción basada en ISO 17100]. 译苑新谈》. New Perspectives In Translation Studies, (8): 94-101.

Díaz Fouces, Oscar. 2019. "Algunas consideraciones sobre el papel de las tecnologías en los Estudios de Traducción y en la formación de traductores». Hikma, 18 (1): 57-84.

EUATC. 2019. «Expectations and Concerns of the European Language Industry». Acceso el 15 junio 2020. https://euatc.org/wp-content/uploads/2019/11/2019-Language-Industry-Survey-Report.pdf

GE, Chang. 2018. «上海高校CAT课程设置与教学情况调查 [Investigación del currículo TAO y situación docente en las universidades de Shanghai)]». Trabajo Fin de Máster. Universidad de China Oriental de Ciencia Política y Derecho.

KRÜGER, Ralph. 2016. «Contextualising Computer-Assisted Translation Tools and Modelling Their Usability ». Trans-kom 9 [1] (2016): 114-148.

LI, Xianling. 2012. 《高校计算机辅助翻译 (CAT) 技术的教学现状调查及其改进策略研究 [Investigación sobre el estado de la enseñanza de la tecnología de traducción asistida por ordenador (CAT) en las universidades y sus estrategias de mejora] ". Trabajo Fin de Máster. Universidad de Estudios Internacionales de Shanghai.

LIu, Lixiang. 2017. «英语专业学习者 CAT 工具使用调查及其教学启示 [Encuesta sobre el uso de herramientas CAT por parte de los estudiantes de la carrera inglesa y sus implicaciones para la enseñanza]». 集美大学学报 [Jimei Daxue Xuebao], 82-88.

MaHFouz, Imán. 2018. «Attitudes to CAT Tools: Application on Egyptian Translation Students and Professionals». Arab World English Journal (AWEJ) Special Issue on CALL (4).

Muñoz Martín, Ricardo. 2010. "On Paradigms and Cognitive Translatology». En Translation and Cognition, editado por Gregory M. Shreve y Erik Angelone, 169-187. Ámsterdam/Filadelfia: John Benjamins.

Olalla-Soler, Christian. 2013. «Traducción y tecnología: herramientas del proceso traductor como actividad profesional. El punto de vista de los estudiantes». Tradumática, 13: 623- 640.

QIAN, Duoxiu. 2011. 计算机辅助翻译 [La traducción asistida por ordenador]. Beijing: 外语教学与 研究出版社 [Waiyu Jiaoxue yu Chuban Yanjiushe].

SANZ VILLA, Sara. 2015. «El traductor ante la traducción asistida por ordenador y la traducción automática con posedición: estudio comparado de actitudes hacia su uso, necesidad y evolución». Trabajo Fin de Máster. Universidad de Valladolid.

SONG, Xingke, Zhang PINGLI y Cheng YUE. 2011. 《本科英语专业计算机辅助翻译教学中学习动 机与需求调查研究 [Investigación sobre la motivación y la demanda del aprendizaje en la enseñanza de la traducción asistida por ordenador para estudiantes de la carrera inglés]». 皖西学院学报 Wanxi Xueyuan Xuebao], 27 (03): 44-46.

YAO,Yunlei y Zhao XAIOBING. 2017. 《国内计算机辅助翻译综述 [Revisión de la traducción asistida por ordenador en China]». 首都师范大学学报 (自然科学版) [Journal of Capital Normal Univerisy «Natural Science Edition»], (6): 20-25.

ZHou, Xinghua. 2013. «计算机辅助翻译协作模式探究 [Investigación sobre el modelo de cooperación en traducción asistida por ordenador]». 中国翻译 [Zhonguo Fanyi], (2): 77-80.

ZHU, Yubin y Chen XIAOQIAN. 2013. 《国内外四种常见计算 机辅助翻译软件比较研究 [Estudio comparativo de cuatro programas comunes de traducción asistida por ordenador en China y en el extranjero]». 外语电化教学 Maiyu Dianhua Jiaoxue], (149): 69-75.

ZHANG, Chengzhi y Wang HUASHu. 2016. «论翻译学的技术转向 [Sobre el giro técnico de los estudios de traducción]». 翻译界 [Fanyi Jie ], (02): 104-118.

Carmen VALERO-GARCÉS y Yanping TAN Análisis de las actitudes de estudiantes de traducción hacia las herramientas TAO...
CLINA

vol. 6-1, June 2020, 69-88

eISSN: 2444-1961

Ediciones Universidad de Salamanca - CC BY-NC-ND 